Karla Bastos Guedes

\title{
Simulação Eficiente do Sistema Elétrico de Potência Utilizando Filtragem Digital Multitaxa
}

\author{
Tese de Doutorado \\ Tese apresentada ao Departamento de Engenharia \\ Elétrica da PUC-Rio como parte dos requisitos para \\ obtenção do Título de Doutor em Ciências em \\ Engenharia Elétrica.
}

Orientador: Jacques Szczupak

Co-orientador: Sebastião E. M. de Oliveira

Rio de Janeiro

Setembro de 2002 


\title{
Karla Bastos Guedes
}

\section{Simulação Eficiente do Sistema Elétrico de Potência Utilizando Filtragem Digital Multitaxa}

\author{
Tese apresentada como requisito para a obtenção do grau de Doutor pelo \\ programa de Pós-Graduação em Engenharia Elétrica do Departamento de \\ Engenharia Elétrica do Centro Técnico Científico da PUC-Rio. \\ Aprovada pela Comissão Examinadora abaixo assinada. \\ Prof. Jacques Szczupak \\ Orientador \\ Departamento de Engenharia Elétrica - PUC-Rio
}

Prof. Carlos Augusto Duque

UFJF

Prof. Glauco Nery Taranto

COPPE/UFRJ

Prof. Julio Cesar Stachinni de Souza

UFF

Prof. Djalma Mosqueira Falcão

COPPE/UFRJ

Prof. Antonio Carlos Siqueira de Lima

EE/UFRJ

Prof. Ney Augusto Dumont

Coordenador Setorial do Centro

Técnico Científico - PUC-Rio

Rio de Janeiro, 30 de setembro de 2002. 
Todos os direitos reservados. É proibida a reprodução total ou parcial do trabalho sem autorização da universidade, da autora e dos orientadores.

\section{Karla Bastos Guedes}

Graduou-se em Engenharia Elétrica na Universidade Federal Fluminense em 1988. Concluiu o Mestrado em Engenharia Elétrica com ênfase em Sistemas Elétricos de Potência na PUC-Rio em 1996. É professora do Departamento de Geometria do Instituto de Matemática da Universidade Federal Fluminense.

Ficha Catalográfica

Guedes, Karla Bastos

Simulação eficiente do sistema elétrico de potência utilizando filtragem digital multitaxa / Karla Bastos Guedes; orientador: Jacques Szczupak; co-orientador: Sebastião E. M. de Oliveira. - Rio de Janeiro : PUC, Departamento de Engenharia Elétrica, 2002.

[15], 110 f. : il. ; $30 \mathrm{~cm}$

Tese (doutorado) - Pontifícia Universidade Católica do Rio de Janeiro, Departamento de Engenharia Elétrica.

Inclui referências bibliográficas.

1. Engenharia elétrica - Teses. 2. Simulador. 3. Redes elétricas lineares. 4. Filtragem digital multitaxa. I. Szczupak, Jacques. II. Oliveira, Sebastião E. M. de. III. Pontifícia Universidade Católica do Rio de Janeiro. Departamento de Engenharia Elétrica. IV. Título. 
"Três paixões, simples porém irresistivelmente fortes, governaram a minha vida: o desejo de amar, a busca do conhecimento e uma compaixão insuportável pelo sofrimento da humanidade".

Bertrand Russel

"O progresso da ciência nada significa enquanto existirem crianças infelizes".

Albert Einstein

"Eu sustento que a única finalidade da ciência é aliviar a miséria da existência humana".

Berthold Bretch 
Aos meus queridos filhos

Renata, Vicente e Theo. 


\section{Agradecimentos}

A Deus, minha família, meus amigos e todos que direta ou indiretamente contribuíram para a realização deste trabalho, em especial:

Ao professor orientador Jacques Szczupak, pelas idéias, estímulo, compreensão e toda a colaboração e orientação que tornaram viáveis a realização deste trabalho.

Ao professor co-orientador Sebastião E. M. de Oliveira, pelo apoio oferecido durante o desenvolvimento deste trabalho.

À querida professora Leontina, pelas sugestões nas escolhas do orientador e do co-orientador.

À PUC-Rio, pela bolsa de isenção concedida.

Aos professores do Departamento de Geometria da UFF, que tornaram possível a minha dedicação exclusiva a esta pesquisa.

Aos funcionários da secretaria de pós-graduação do DEE da PUC-Rio, Ana, Alcina, Mário e Márcia, pela ajuda, amizade e carinho que sempre ofereceram.

À amiga Silvana Faceroli, pela colaboração e excelente parceria em momentos muito importantes deste trabalho.

Aos professores que participaram da banca, Antonio Carlos, Júlio César, Carlos Duque, Glauco e Djalma, pelos enriquecedores comentários e sugestões apresentadas.

Ao amigo Marco Antônio Cetale e todos os colegas da PUC-Rio.

Aos meus pais, Luiz Fernando e Maria Auxiliadora e à minha avó Florinda, merecedores da minha eterna gratidão, por tudo.

E finalmente, a todos os professores e mestres que a vida me ofereceu. 


\section{Resumo}

Guedes, Karla Bastos; Szczupak, Jacques (Orientador); Oliveira, Sebastião E. M. de (Co-orientador). Simulação Eficiente do Sistema Elétrico de Potência Utilizando Filtragem Digital Multitaxa. Rio de Janeiro, 2002. 125p. Tese de Doutorado - Departamento de Engenharia Elétrica, Pontifícia Universidade Católica do Rio de Janeiro.

Esta pesquisa propõe a implementação de um simulador para investigar o comportamento dinâmico dos sistemas elétricos de potência e a sua estabilidade. É introduzida uma nova abordagem para simulação de redes elétricas lineares, que segue as técnicas básicas e os modelos utilizados no EMTP (simulador tradicional), porém levando vantagem ao utilizar propriedades de filtragem digital multitaxa. O simulador proposto decompõe o sinal e a rede em sub-bandas de freqüência, cada uma delas operada independentemente, o que torna o processo bastante interessante para a implementação utilizando processamento em paralelo. O passo de integração nas simulações das sub-bandas é sempre maximizado, reduzindo a carga computacional. A simulação permite a detecção em tempo real de sub-bandas que poderiam ser momentaneamente desconectadas, sem perda de precisão no resultado final. Este comportamento adaptativo aumenta a eficiência do simulador, ajustando a complexidade do modelo de acordo com os requisitos da simulação.

\section{Palavras-chave}

Simulador, redes elétricas lineares, filtragem digital multitaxa. 


\section{Abstract}

Guedes, Karla Bastos; Szczupak, Jacques (Advisor); Oliveira, Sebastião E. M. de (Advisor). Efficient Simulation of the Electric Power System using Multirate Digital Filtering. Rio de Janeiro, 2002. 125p. DSc. Dissertation - Departamento de Engenharia Elétrica, Pontifícia Universidade Católica do Rio de Janeiro.

This work proposes the implementation of a simulator to investigate the dynamic behavior and the stability of electric power systems. A new approach to linear electric network simulation is introduced, closely following the EMTP basic techniques and models, but taking advantage of multirate digital filter properties. The final simulator decomposes signals and network models into subbands of frequencies, each of them independently operated, allowing parallel processing The integration time step in the subband simulations is always maximized, reducing computational burden. The simulation permits online detection of subbands that could be momentarily disconnected with negligible loss of accuracy in the final result. This adaptive feature further improves efficiency, adjusting the network model complexity, according to the simulation requirements.

\section{Keywords}

Simulator, linear electrical networks, multirate digital filtering. 


\section{Sumário}

$\begin{array}{ll}\text { Lista de Figuras } & 12\end{array}$

Lista de Tabelas $\quad 15$

1. Introdução 16

1.1. Considerações Gerais 16

$\begin{array}{ll}\text { 1.2. Objetivos e estrutura da tese } & 18\end{array}$

2. O simulador tradicional 20

2.1. Introdução 20

2.2. Topologia de redes elétricas $\quad 21$

2.3. Alguns conceitos básicos de teoria de grafos lineares 21

2.3.1. Grafo linear 21

2.3.2. Subgrafo 22

2.3.3. Grafo orientado e não-orientado 22

2.3.4. Caminho 22

2.3.5. Grafo conexo 23

2.3.6. Circuito 23

2.3.7. Árvore 23

2.3.8. Corte 24

2.3.9. Matriz Incidência $\quad 24$

2.4. Modelagem analógica da rede elétrica 25

2.5. Modelagem digital da rede elétrica 28

2.5.1. Modelo digital do elemento linear a parâmetro concentrado $L \quad 29$

2.5.2. Modelo digital do elemento linear a parâmetro concentrado C $\quad 30$

2.5.3. Modelo digital do elemento linear a parâmetro concentrado R 31

2.5.4. Modelo digital do ramo geral 31

2.5.5. Modelo digital de redes elétricas monofásicas 32

2.5.6. Modelo digital de redes elétricas trifásicas 36

2.6. Exemplos de modelagem e simulação 37

2.6.1. Simulação de redes elétricas monofásicas - Sistema-teste 138 
2.6.2. Simulação de redes elétricas trifásicas - Sistema-teste

3. Conceitos básicos de filtragem digital multitaxa 45

3.1. Introdução 45

3.2. Filtros digitais $\quad 45$

3.2.1. Estrutura básica de filtros digitais FIR 46

3.2.2. Estrutura básica de filtros digitais IIR 47

3.3. O decimador 48

3.4. O expansor ou interpolador 50

3.5. Equivalências e Identidades nobres 52

3.6. Bancos de filtros digitais 53

3.7. Bancos de filtros de reconstrução perfeita 55

3.8. Exemplos de bancos de filtros 58

$\begin{array}{ll}\text { 3.9. Conclusões } & 61\end{array}$

4. O simulador proposto 62

4.1. Introdução 62

4.2. Modelagem digital da rede elétrica em sub-bandas de freqüência 63

4.3. Os simuladores multitaxa 65

4.4. Processo automático para alteração da taxa de amostragem 69

4.5. Processo automático de comutação entre os simuladores 73

4.6. Simulador multitaxa para sinais de faixa estreita de freqüência 74

4.7. Exemplos ilustrativos 82

4.7.1. Sistema-teste $3 \quad 82$

4.7.2. Sistema-teste $4 \quad 88$

4.8. Conclusões 91

5. Modelagem da máquina síncrona e seus controles associados 92

5.1. Introdução 92

5.2. Princípio de funcionamento dos geradores síncronos 92

5.3. Modelagem da máquina síncrona 93

$\begin{array}{ll}\text { 5.3.1. Modelagem elétrica } & 94\end{array}$ 
5.3.2. Modelagem do sistema mecânico

5.4. Modelagem do sistema de controle de tensão da máquina síncrona

5.4.1. Controle de tensão do tipo a 98

5.4.2. Controle de tensão do tipo b 99

5.5. Modelagem do sistema de controle de velocidade da máquina $\begin{array}{ll}\text { síncrona } & 100\end{array}$

5.6. Inserção das equações discretizadas do gerador síncrono nos simuladores multitaxa

5.7. $\quad$ Esquema de predição 105

5.8. Oscilações numéricas nos resultados das simulações 105

5.8.1. A transformação bilinear 106

5.8.2. Equivalência entre a transformação bilinear e a regra de $\begin{array}{ll}\text { integração trapezoidal } & 106\end{array}$

5.8.3. Procedimento para correção das oscilações numéricas 107

5.9. Exemplos llustrativos 107

$\begin{array}{lll}\text { 5.9.1 Sistema-teste } 5 & 108\end{array}$

5.10. Conclusões 113

6. Comparação de carga computacional 115

6.1. Introdução 115

6.2. Tabelas comparativas 115

$\begin{array}{ll}\text { 6.3. Conclusões } & 117\end{array}$

7. Conclusões 118

7.1. Principais contribuições 119

7.2. Sugestões para trabalhos futuros 119

8. Referências bibliográficas 121

Apêndice A. Dados dos geradores síncronos e de seus sistemas de controle 


\section{Lista de figuras}

Figura (2.1) - (a) Circuito; (b) Grafo correspondente 21

Figura (2.2) - (a) Grafo orientado;(b) Grafo não-orientado. 22

Figura (2.3) - Modelo analógico do ramo geral. 25

Figura (2.4) - Modelo discreto do indutor. 28

Figura (2.5) - Modelo discreto do capacitor. 30

Figura (2.6) - Modelo discreto do ramo geral. 31

Figura (2.7) - Sistema-teste 1.

Figura (2.8) - Grafo associado ao Sistema-teste 1.

Figura (2.9) - Corrente no ramo 3 do Sistema-teste 1.

Figura (2.10) - Tensão 6 na barra 3 do Sistema-teste 1.

Figura (2.11) - Sistema-teste 2.

Figura (2.12) - Grafo associado ao Sistema-teste 2. 41

Figura (2.13) - Tensão na barra 1 do Sistema-teste 2

Figura (2.14) - Corrente no ramo 5 do Sistema-teste 2.

Figura (3.1) - Filtro digital. $\quad 46$

Figura (3.2)- Exemplo de estrutura de filtros digitais FIR de terceira
ordem.

Figura (3.3) - Exemplo de estrutura não canônica de filtros digitais

IIR de terceira ordem. $\quad 47$

Figura (3.4) - Bloco operador decimador por M antecedido por filtro. $\quad 48$

Figura (3.5) - Demonstração de decimação no domínio do tempo $M=2$. 48

Figura (3.6) - Ilustração de decimação no domínio da freqüência

(sem a ocorrência de superposição ou "aliasing"), M=2. 49

Figura (3.7) - Ilustração de decimação no domínio da freqüência

(com a ocorrência de superposição ou "aliasing"), M=3.

Figura (3.8) - Bloco expansor por $L$ seguido de filtro seletivo. $\quad 50$

Figura (3.9) - Demonstração do expansor no domínio do tempo, L=2 51

Figura (3.10) - Ilustração do expansor no domínio da freqüência, L=2 51

Figura (3.11) - Equivalências ou identidades simples utilizadas em

sistemas multitaxa. $\quad 52$

Figura (3.12) - Identidades nobres utilizadas em sistemas multitaxa. $\quad 53$

Figura (3.13) - (a) Banco de filtros de análise; (b) Banco de filtros de síntese.

Figura (3.14) - Representação de filtragem multitaxa por árvore binária 54

Figura (3.15) - Representação por componentes polifásicos. 54

Figura (3.16) -Sinal de entrada $x(n)$ e sinal reconstruído. 59

Figura (3.17) - Atraso do sinal reconstruído em relação ao sinal de
entrada $x(n)$.

Figura (3.18) - Superposição dos sinais $x(n)$, e erro. 60

Figura(3.19)- Exemplo de filtragem por banco de reconstrução perfeita 61

Figura (4.1) - Simulador com um nível de decomposição em

sub-bandas. 
Figura (4.2) - Simulador com dois níveis de decomposição em subbandas. Decomposição não uniforme.

Figura (4.3) - Simulador com três níveis de decomposição em subbandas. Decomposição não uniforme.

Figura (4.4) - Diagrama de blocos das equações inseridas em simulador com um nível de decomposição.

Figura (4.5) - Equações (4.7) e (4.8) escritas em forma de diagrama de blocos.

Figura (4.6) - Exemplo de decimação em banco de filtros do tipo árvore binária.

Figura (4.7) - Processo automático para desativar sub-banda. $\quad 70$

Figura(4.8)- Energia do sinal de alta freqüência.

Figura(4.9) - Simulação multitaxa que utilizou o valor de $\xi=0.001$ no processo automático de variação da taxa de amostragem.

Figura(4.10) - Simulação multitaxa que utilizou o valor de $\xi=0.1$ no processo automático de variação da taxa de amostragem.

Figura(4.11) - Comutação entre os simuladores.

Figura (4.12) - (a) Resposta típica de um filtro causal; (b) Resposta típica de um filtro ideal.

Figura (4.13) - Simulador multitaxa para sinais de faixa estreita de freqüência.

Figura (4.14) - Resultado das decimações por 2 de um sinal de faixa estreita com as frequências analógicas correspondentes indicadas em superposição aos ângulos que traduzem as frequências digitais, relativas à de amostragem.

Figura (4.15) - Efeito da decimação por 2 no no espectro de freqüência de um sinal.

Figura (4.16) - Reconstrução do sinal do simulador multitaxa 7.

Figura (4.17) - Ampliação da região marcada na Figura (4.16).

Figura (4.18) - Comparação entre o espectro em freqüência dos sinais do simulador tradicional e do simulador multitaxa 7.

Figura (4.19) - Resultado da primeira etapa das decimações por 2 de um sinal de faixa estreita.

Figura (4.20) - Resultado da segunda etapa das decimações por 2 de um sinal de faixa estreita.

Figura (4.21) - Diagrama unifilar do sistema-teste 3

Figura (4.22) - Corrente na carga L6 do Sistema-teste 3 utilizando o simulador tradicional e o simulador com 1 nível de divisão em sub-bandas.

Figura (4.23) - Atraso do simulador 1 em relação ao simulador tradicional

Figura (4.24) - Corrente na carga L6 do Sistema-teste 3 utilizando o simulador tradicional e o simulador 2 .

Figura (4.25) - Atraso do simulador 2 em relação ao simulador Tradicional.

Figura (4.26) - Corrente na carga L6 do Sistema-teste 3 utilizando o simulador tradicional e o simulador 3.

Figura (4.27) - Atraso do simulador 3 em relação ao simulador tradicional.

Figura (4.28) - Corrente na carga L6 do Sistema-teste 3. 
Figura (4.29) - Diagrama unifilar do Sistema-teste 4.

Figura (4.30) - Tensão na barra 1 do Sistema-teste 4.

Figura (4.31) - Tensão na barra 1 do Sistema-teste 4, reconstruída. $\quad 89$

Figura (4.32) - Ampliação da região marcada na Figura (4.31). 90

Figura (5.1) - Representação mecânica da máquina síncrona. 93

Figura (5.2) - Representação elétrica da máquina síncrona. 94

Figura (5.3) - Representação dos circuitos de eixos d e q do modelo utilizado.

Figura (5.4) - Representação do controle de tensão do tipo a da máquina síncrona.

Figura (5.5) - Representação do controle de tensão do tipo b da máquina síncrona.

Figura (5.6) - Representação do controle de velocidade da máquina síncrona.

Figura (5.7) - Equações que compõem o bloco Gerador. 102

Figura (5.8) - Simulador com um nível de decomposição em sub-bandas.

Figura (5.9) - Simulador com dois níveis de decomposição em sub-bandas.

Figura (5.10) - Simulador com três níveis de decomposição em sub-bandas.

Figura (5.11) - Simulador multitaxa para sinais de faixa estreita com $\mathrm{M}$ níveis de decomposição em sub-bandas.

Figura (5.13) - Corrente no capacitor 3 do Sistema-teste 5.

Figura (5.14) - Tensão na barra 2 do Sistema-teste 5.

Figura (5.15) - Corrente no gerador 1, do Sistema-teste 5. 110

Figura (5.16) - Tensão reconstruída da barra 1, do Sistema-teste 5. 112

Figura (5.17) - Ampliação da região marcada na Figura (5.16). 113 


\section{Lista de Tabelas}

Tabela (2.1) - Transformadas de Laplace aplicadas à Eq. (2.3). 27

Tabela (4.1) - Definição das matrizes apresentadas na Figura (4.4). 67

Tabela (4.2) - Valores dos pontos P1 e P2 mostrados na Figura (4.5). 68

Tabela (4.3) - Comparação da carga computacional - Sistema-teste 3. 87

Tabela (4.4) - Comparação da carga computacional - Sistema-teste 4. 90

Tabela (6.1) - Redução de carga computacional dos simuladores multitaxa. Sistemas-teste 1, 4 e 5.

Tabela (6.2) - Redução de carga computacional dos simuladores Multitaxa. Sistema-teste 2.

Tabela (6.3) - Redução de carga computacional dos simuladores Multitaxa. Sistema-teste 3.

Tabela (6.4) - Redução de carga computacional dos simuladores Multitaxa. Sistema com 3600 ramos. 